\title{
Review Article \\ Searches for Dark Matter with Superheated Liquid Techniques
}

\author{
A. Pullia \\ Department of Physics and I.N.F.N., University of Milano-Bicocca, Piazza della Scienza 3, 20135 Milano, Italy \\ Correspondence should be addressed to A. Pullia; antonino.pullia@mib.infn.it \\ Received 5 March 2014; Accepted 25 April 2014; Published 12 June 2014 \\ Academic Editor: Anselmo Meregaglia
}

Copyright (c) 2014 A. Pullia. This is an open access article distributed under the Creative Commons Attribution License, which permits unrestricted use, distribution, and reproduction in any medium, provided the original work is properly cited. The publication of this article was funded by SCOAP ${ }^{3}$.

This is a short review of the detectors based on the superheated liquid techniques, including continuously sensitive bubble chambers, superheated droplet detectors (SDD) and Geysers.

\section{Introduction}

One of the most celebrated detectors operating at accelerators is the bubble chamber [1]; very important discoveries were done employing this technology during the sixties and seventies.

Bubble chambers were divided into two categories (hydrogen and heavy liquid bubble chambers); the former ones (like the " $80 \mathrm{~cm}$," BEBC, 15-foot Flab, Argonne 30 inches, etc.) had the advantage that the target was well defined and static; the latter ones (Gargamelle, BP3, 15-foot Bubble Chamber, SKAT, etc.) had a bigger stopping power and were particularly suited to identify the nature of the secondary produced particles like electrons, gamma rays, and pions and kaons decays. Many discoveries were done by bubble chambers: several resonances, the neutral currents, leptonic and semileptonic, the $\Omega^{-}$, and so forth.

Their use decreased with the birth of the "electronic detectors" capable of performing automatic event selection and scanning and collecting and analysing much more events.

However the expansion of the bubble chamber was linked to the beam passage: the switching off of the acceleration of the primary particles was used to command it.

So the bubble chambers were commanded by the beam passage and the used liquid reached a metastable equilibrium state which occurs when the pressure of the liquid was lowered adiabatically: the substance remains in the liquid state despite the vapor pressure or the boiling point temperature.

The metastability of the liquid makes it possible to detect charged particles. When the liquid is brought to a temperature and pressure, where, according to its phase diagram, it should be gaseous but maintains the liquid phase, it is said to be "superheated." The difference in pressure between the vapor pressure and the operating pressure of a bubble chamber is known as "degree of superheat."

The higher this degree is, the less stable the liquid is, but at high degree of superheat the bubble chamber becomes more sensitive to lower energy particles that can interact with the nuclei giving lower energy recoils and becomes sensitive to electrons, $\gamma$ rays, high energy muons, and so forth.

These particles are an important background for the search of dark matter, so, in order to exploit superheated detectors for direct detection of dark matter, the operation technique had to be changed [2-4].

\section{Bubble Nucleation in Superheated Liquids}

The phenomena describing the formation of a bubble in a superheated Liquid are the nucleation and the growing of the bubble.

The nucleation and the growing are described by the theory of Seitz [5-7] which is briefly summarized in the following.

A charged particle loses energy along its trajectory through a superheated liquid via ionization, collision, and radiation.

Thus the primary particle leads to a temporary thermal excitation along its track; the temperature of the gas created is hotter than that of the surrounding liquid. The Seitz model is named as "hot spike" model of bubble nucleation. 
If the pressure of the hot gas is sufficient, a protobubble will overcome the surface tension and the bubble grows. Its growth is due to its internal pressure which is the vapor pressure of the liquid at the current temperature $P_{v}$ (this pressure is greater than the pressure outside the bubble $P_{e}$ by definition of superheated liquid); in this case the bubble becomes visible. To reach this condition the radius of the protobubble must be as follows:

$r_{c}>(2 \lambda) /(\Delta P)$, where $\Delta P=P_{v}-P_{e}$ and $\lambda$ is the surface tension of the liquid. Furthermore the stopping power must be $d E / d x>E_{c} /\left(2 r_{c}\right)$, where

$$
E_{c}=4 \pi r_{c}^{2}\left(\lambda-\frac{T d \lambda}{d T}\right)+\left(\frac{4 \pi r_{c}^{3}}{3}\right)\left(\left(\frac{\rho_{v} h_{f g}}{M}\right)-\Delta P\right),
$$

$\rho_{v}$ is the saturated vapor density, $h_{f g}$ is the latent heat of vaporization per mole, and $M$ is the molecular mass [8].

If $E<E_{c}$ and the relation $d E / d x>E_{c} /\left(2 r_{c}\right)$ is not fulfilled, $r<r_{c}$ and then the protobubble created is smaller than the critical radius; it will collapse and disappear.

\section{Application of Superheated Devices to WIMP Searches}

To be useful as dark matter detectors the bubble formation devices needed several changes to fulfil three important constraints:

(i) to be more stable than traditional high energy physics bubble chamber (reaching a quasicontinuously sensitive operation);

(ii) to be triggered when a dark matter particle crosses the detector and interacts with it;

(iii) to have a strong rejection of the principal backgrounds that can simulate a dark matter interaction with the ordinary matter. The rarity of the interactions also changes the nature of the bubble devices from that of a tracking device (full of multiple tracks of small bubbles from different particle crossing the detector) to a counting device.

This different way to use a bubble detector as a counting detector brought the interested physicists in three directions:

(1) new type of bubble chambers;

(2) SDD which are superheated droplet detectors;

(3) the Geyser detectors.

In this paper I concentrate on the three directions mentioned above and I summarize the most relevant results and the proposals for the future.

In the following, I will focus on weakly interacting massive particle (WIMP) as the most plausible candidate for dark matter.

WIMPs interact not only with gravitational fields but also weakly; in this case indeed the search of their direct interaction is not without hope. Many experimental methods have been studied and realized to detect directly WIMPs. They include the use of scintillators $\mathrm{NaI}$ [9], liquid argon [10], xenon chamber [11], cryogenic semiconductors [12], and detectors based on the nucleation of bubbles [2-4]. The results obtained with these detectors are in some case in contradiction and need a supplement of work to make clear the situation; the development of alternative and complementary techniques is thus particularly motivated.

\section{Bubble Chamber}

The experiments with bubble chambers are concentrated on the work of the Collaboration COUPP (Chicagoland Observatory for Underground Particle Physics).

4.1. $2 \mathrm{~kg}$ Chamber (1L) Filled with $\mathrm{CF}_{3} I$ (Experiment T945). The first dark matter limits SD $[13,14]$ produced by COUPP was achieved with a $2 \mathrm{~kg}(1 \mathrm{~L})$ prototype which produced the best spin-dependent (SD) proton limits at the time over a significant mass range. This chamber was built at the University of Chicago and tested at the Laboratory for Astrophysics and Space Research (LASR) at a depth of six m.w.e.; the results are reported in Figure 1.

4.2. Modified $2 \mathrm{~kg}$ Chamber (1L). Due to the very high background from Radon filtering an O-ring, the first version of the chamber was modified: substitution of the O-ring and replacement of the quartz jar with a new, acid-etched, and precision cleaned jar; data were taken in NUMI (NeUtrinos at Main Injector) at Fermilab.

4.3. $4 \mathrm{~kg}$ Chamber (2 L) (Figure 2 and $[13,14])$. This chamber worked in two phases which are

(a) filled with $\mathrm{CF}_{3} \mathrm{I}$;

(b) filled with $\mathrm{C}_{3} \mathrm{~F}_{8}$;

phase (a): the preliminary results are reported in the talk of Ardid at Trieste [15, 16] (see Figure 3). phase (b): $4 \mathrm{~kg}$ filled with $\mathrm{C}_{3} \mathrm{~F}_{8}$.

Excellent sensitivity was obtained for low energy recoils $(3 \mathrm{keV})$ at SNOLAB $[15,16]$, but this phase is in progress and no definitive results where reported up to now.

4.4. The Big Bubble Chamber $(30 \mathrm{~L}=60 \mathrm{~kg})$ [17]. This chamber is working in SNOLAB filled at the moment with $37 \mathrm{~kg}$ of $\mathrm{CF}_{3} \mathrm{I}$; the installation was completed in June 2013; a run collecting $50000 \mathrm{~kg}$-days data is foreseen in the future, with a possible increase of the detector's mass.

No results have been yet reported for the moment; the sensitivity of this chamber is shown in Figure 4.

4.5. Proposal for a $500 \mathrm{~kg}$ Bubble Chamber. For the future the collaboration PICO (which joints the expertise of COUPP 


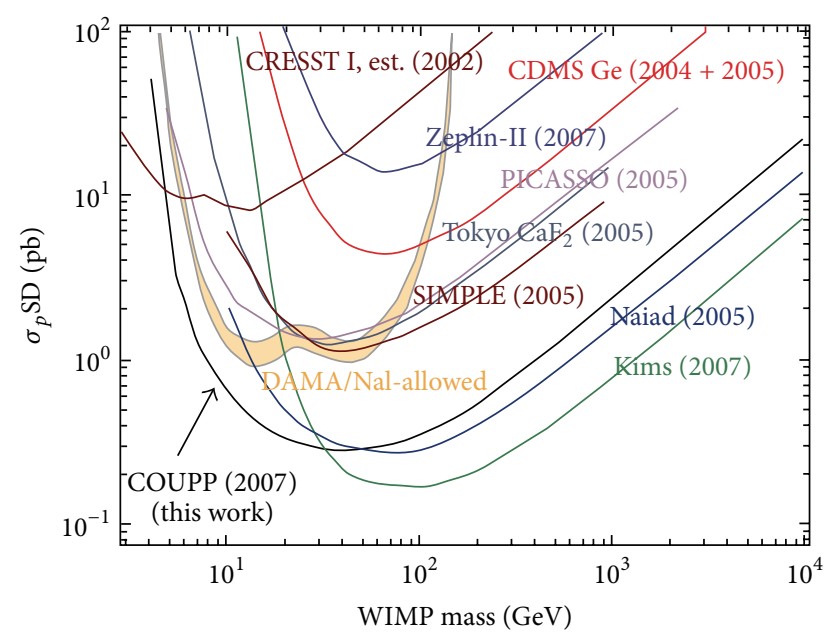

(a)

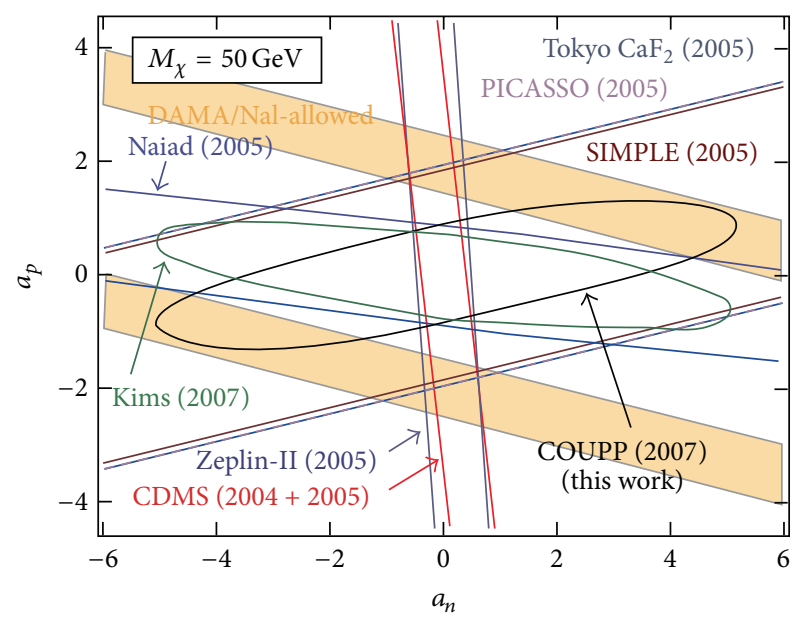

(b)

Figure 1: COUPP, $2 \mathrm{~kg}$ bubble chamber: (a) limits (90\% C.L.) on spin-dependent (SD) WIMP-proton cross section (picobarns) from COUPP; (b) similar limits for the SD parameters $a_{p}$ and $a_{n}$.

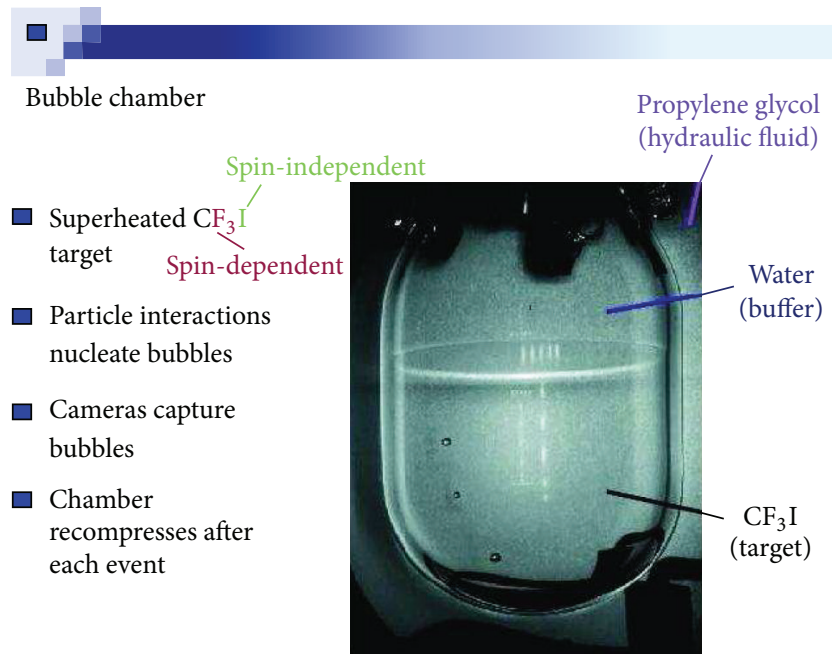

Figure 2: Peter Cooper CERN PH Seminar on July 10, 2012: the $4 \mathrm{~kg}$ COUPP bubble chamber. and PICASSO) plans to build a new bubble chamber on the scale of tons [18].

The conceptual design is well developed. If the results from COUPP- 4 and COUPP- 60 are scaled up, the expected sensitivities are reported in Figures 5 and 6 for a filling of $\mathrm{C}_{3} \mathrm{~F}_{8}$.

\section{Superheated Droplet Detectors}

Superheated droplet detectors (SDD) are also based on the technique of the superheated bubble formation.

In contrast to bubble chambers used in high energy physics, which are based on the same principle, SDD are basically continuously sensitive, since one droplet at a time undergoes phase transition. Only occasionally, for instance, every few days the detector medium is set under pressure in order to transform gas bubbles back to liquid droplets.

The rupture of metastability by radiation has been used as a method in particle detection. The most important application was the bubble chamber. Apfel [19] extended this concept in the form of SDD in which small drops of the superheated liquid are uniformly dispersed in a gel or viscoelastic medium: it isolates the fragile metastable system from vibrations and convections currents that occur in bubble chambers; in Figure 7 a sketch of a detector exposed to a neutron flux is shown.

The lifetime of the superheated state becomes very long, allowing applications of the SDD as neutron dosimeters and detectors for dark matter.

Two experiments have used SDD for searches of direct interaction of WIMP with ordinary matter: SIMPLE and PICASSO.

SIMPLE is superheated instrument for massive particle.

PICASSO is Project in Canada to Search for Supersymmetric Objects.

SIMPLE obtained the first important results (see Figure 8); the limit curve in function of the WIMP mass is shown in $[8,20]$.

For PICASSO, see Figure 9 for technical procedures and [21] for results.

In comparison with the bubble chamber the SDD technique has at least the following advantages:

(1) stability for much longer times;

(2) lower cost $(0.19 \mathrm{k} \$ / \mathrm{kg})$;

(3) much less impurities $(\gamma, \alpha$, and $\beta$ due to the avoided contact with the wall of the vessel and with the buffer liquid),

and the following disadvantages:

(1) very poor quantity of sensitive matter (maximum $3 \%$ of the gel). This makes impossible a competition with the proposals at the ton scale for different detectors (see Table 1). 


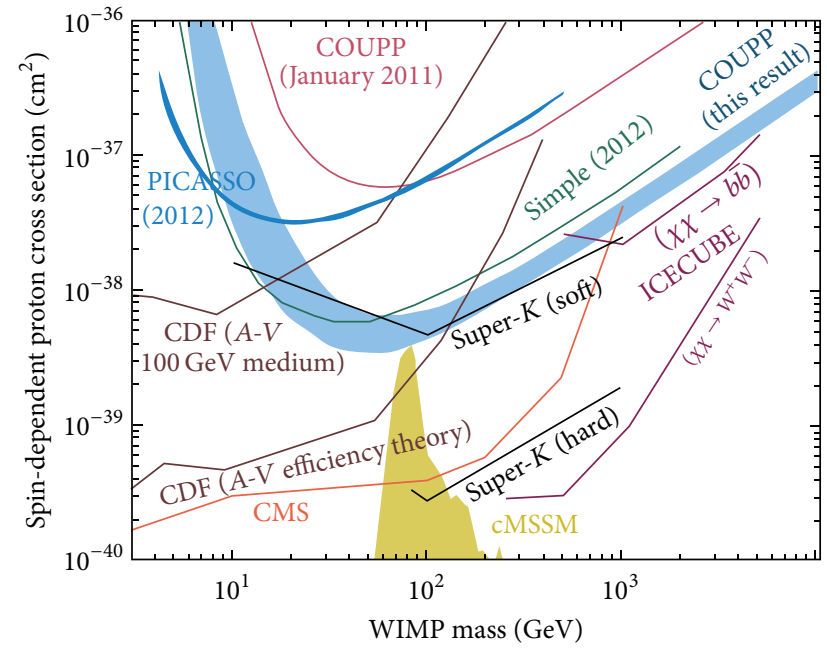

(a)

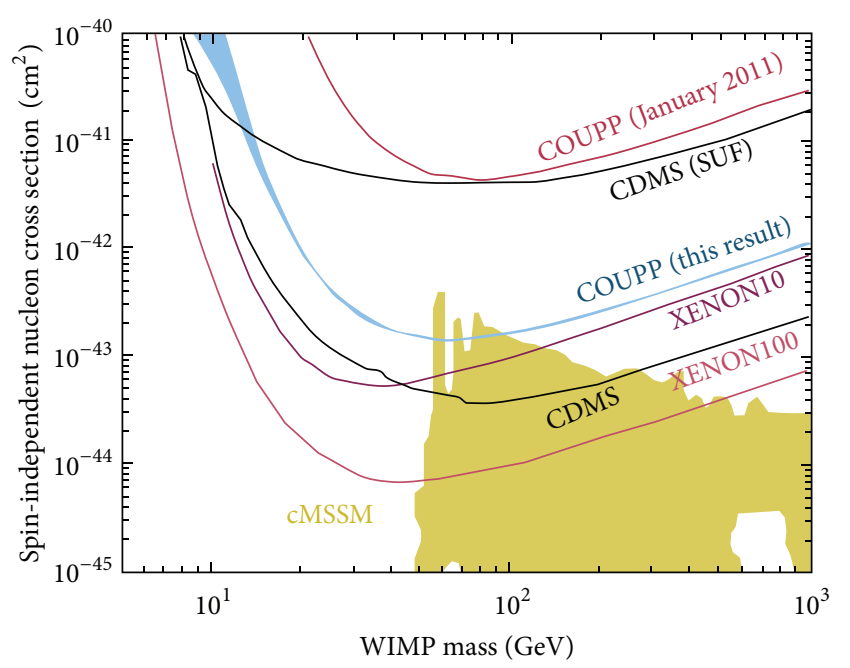

(b)

Figure 3: Preliminary results of the $4 \mathrm{~kg}$ bubble chamber: from Ardid [15], Italy, on August 26, 2013.

TABLE 1: Comparison between different techniques.

\begin{tabular}{lccc}
\hline & SIMPLE (SDD) & PICASSO (SDD) & Bubble chamber (COUPP) \\
\hline Detector construction & House & Industry & House \\
Liquid & $\mathrm{C}_{2} \mathrm{ClF}_{5}$ & $\mathrm{C}_{2} \mathrm{ClF}_{5} ; \mathrm{C}_{4} \mathrm{~F}_{8}$ & $\mathrm{CF}_{3} \mathrm{I}_{3} \mathrm{C}_{3} \mathrm{~F}_{8}$ \\
Installation (m.w.e.) & GESA (1500) & SNOLAB (6010) & LASR (6), NUMI (300), and SNOLAB (6010) \\
Results & Figure 8 & Figure 9 and [21] & Figure 5 \\
\hline
\end{tabular}

\section{Trigger of SDD and Bubble Chambers}

R\&D within SDD detectors brought an interesting feature into light: the sound emitted at the bubble formation [22] is different if the bubble is due to a recoiling nucleus (as happens for an interaction of neutron or WIMP) or if the bubble is induced by an $\alpha$ decay [23].

Energetic charged particles traversing liquids or solids produce acoustic waves during their passage (see ANTARES and ICECUBE $[24,25]$ experiments in the PeV range of energy). However, for processes useful for dark matter (in the range of $10-100 \mathrm{keV}$ ), the emitted sound predicted by the thermoacoustic effect is not detectable. Nevertheless particle interactions in stressed or superheated liquids produce detectable acoustic signal that is characteristic of the nature or the extension of the primary event.

This suggests that the superheated liquids provide an intrinsic amplification mechanism with a gain of $10^{5}$.

In Figure 10 [23] and Figure 11 typical spectra are reported for recoils induced by neutrons from an "Am-Be" source.

Ions from nuclear recoils indeed have ranges with sub- $\mu \mathrm{m}$ length; on the contrary the $\alpha$ emitter (inside the superheated liquid) can provide two sources of ionizations (the $\alpha$ itself with a track length of about $40 \mu \mathrm{m}$ for an energy of $5 \mathrm{MeV}$ and the daughter nucleus.) In Figure 11 [23] such an effect is shown.

The sound signal must be transformed to electronic signals by transducers accompanying the detector, studied with a Fourier analysis and described by an acoustic energy parameter. The success of this possible separation of the $\alpha$ background has quickly stimulated COUPP and this technique was applied to the bubble chambers; the level of the rejection of this background is now $<10^{-3}$, still keeping an acceptance for recoiling nuclei of $98 \%$ [21].

\section{The Geyser or Condensation Chamber}

Two groups are interested in a new technique called "Geyser":

(1) the Milano-Bicocca group MOSCAB [26, 27];

(2) the PICO group [28].

This technique is a variant of the superheated liquid technique of extreme simplicity.

The main volume of the target liquid $\left(\mathrm{C}_{3} \mathrm{~F}_{8}\right.$, e.g., $)$ is kept in a thermal bath at a constant temperature $T_{L}$.

The vapour above the liquid is kept at a temperature $T_{V}<$ $T_{L}$ by cooling the top of the vessel by a circulating liquid (water).

The equilibrium vapour pressure above the liquid is $P_{V}$ so the liquid is in a state of underpressure and therefore a superheat of $\Delta p=P_{V}-P_{L}$, where $P_{V}=P_{\text {Sat. }}\left(T_{L}\right)$ and $P_{L}=P_{\text {Sat. }}\left(T_{V}\right)$. A local energy release of energy due to, for instance, a recoiling ion induced by a WIMP interaction can produce a vapour bubble which can grow (if over a threshold in energy) to visible size. This vapour bubble rises in the liquid and pushes up part of the liquid in the neck (this is the reason of the name Geyser). When equilibrium pressure 


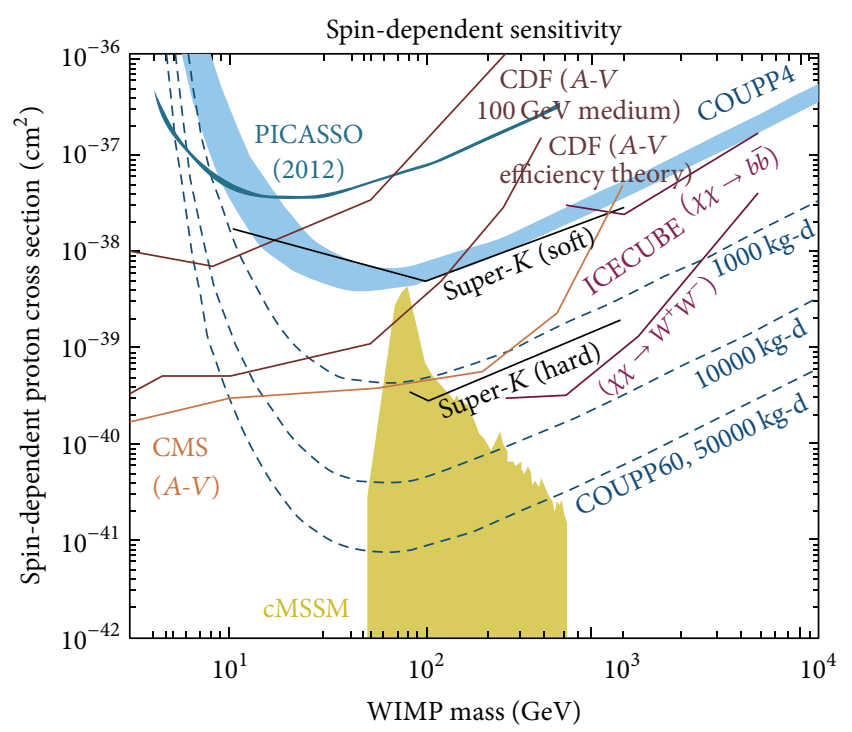

(a)

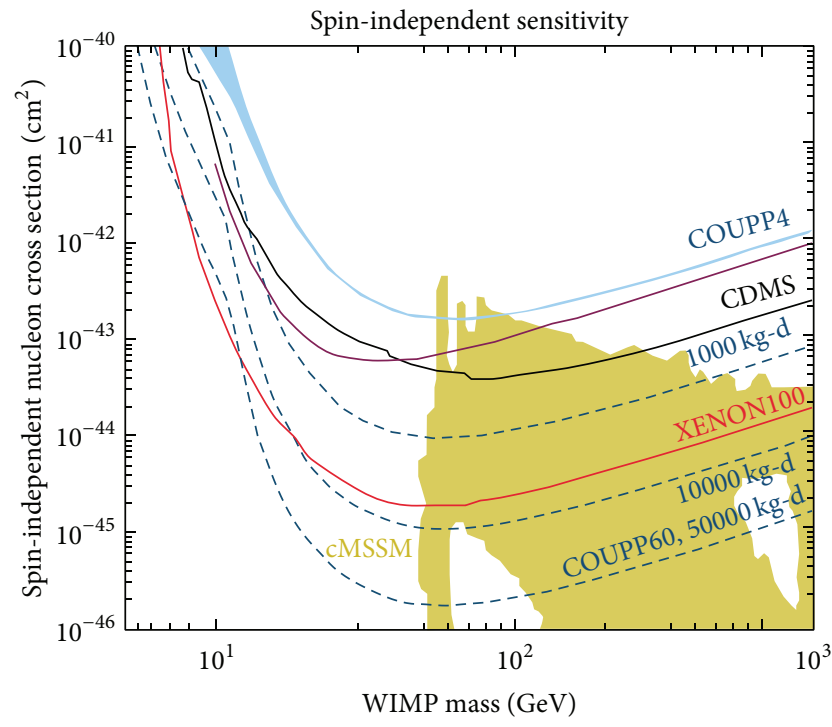

(b)

Figure 4: Sensitivity of the $60 \mathrm{~kg}$ bubble chamber: from Ardid [15], Italy, on August 26, 2013.

is reached, the hot vapour in the top of the vessel recondenses and the liquid is recovered into the main volume. The original metastable state is recovered in a few seconds and the system is ready to record a new event. The system does not require external intervention or recompression.

In Figure 12 a drawing of the principal parts of the MOSCAB Geyser is shown.

The figure represents a vertical section of a cylinder, so the coils used as sources of heat are represented by small circles.

In the top part of the same figure the pressure equalizers are shown; they are constituted by two elastic membranes that push the external water when the pressure of the freon gas increases and acts also in the reverse sense.

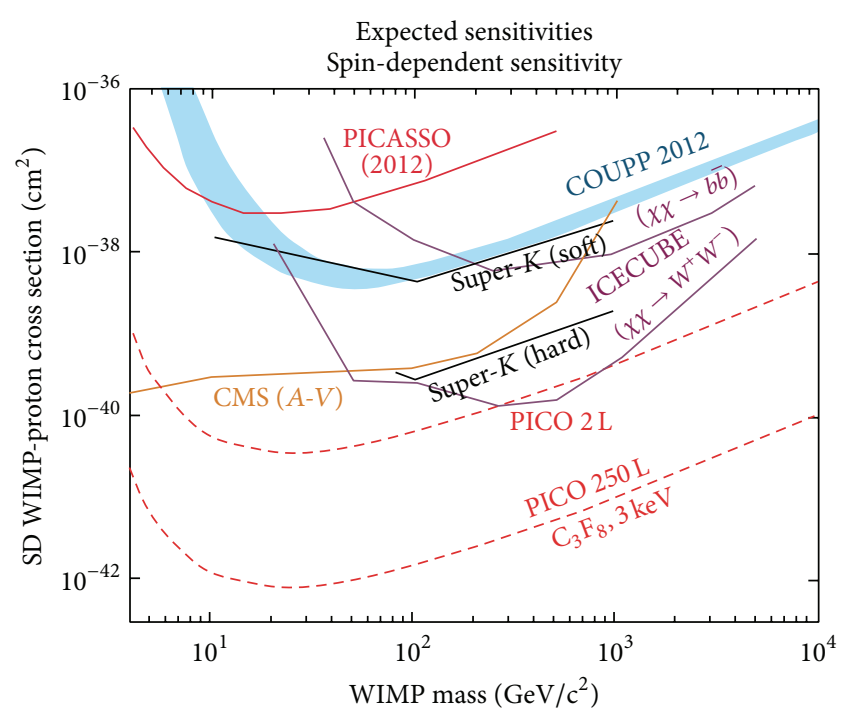

FIgURE 5: Expected sensitivity for the PICO proposal of a $500 \mathrm{~kg}$ detector (SD): from Ardid [15], Italy, on August 26, 2013.

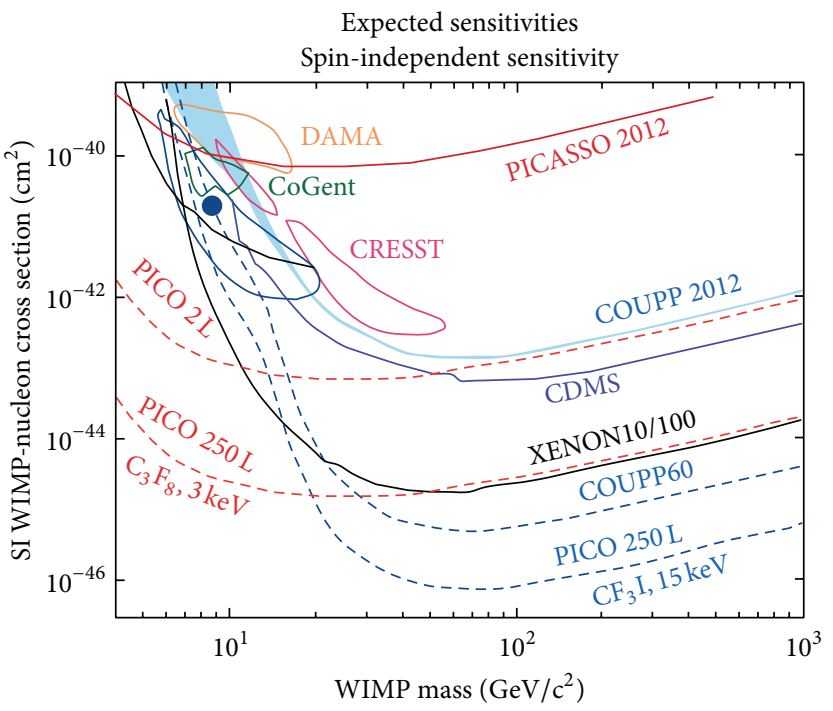

FIGURE 6: Expected sensitivity for the PICO proposal of a $500 \mathrm{~kg}$ detector (SI): from Ardid [15], Italy, on August 26, 2013.

In Figure 13 there is a picture of the apparatus built in Milano. In the bottom the liquid freon is shown; the buffer liquid glycol that separates the liquid freon from the vapour is also shown. The degree of superheat applied must exclude the detection of minimum ionizing particles (electrons and $\gamma$ rays) and on the contrary it must allow the detection with high efficiency of the recoiling ions.

The principal advantages of the Geyser (and of the bubble chamber) are the following.

(1) The strong rejection of the particles at minimum ionization (electrons and $\gamma$ ) is an advantage. 


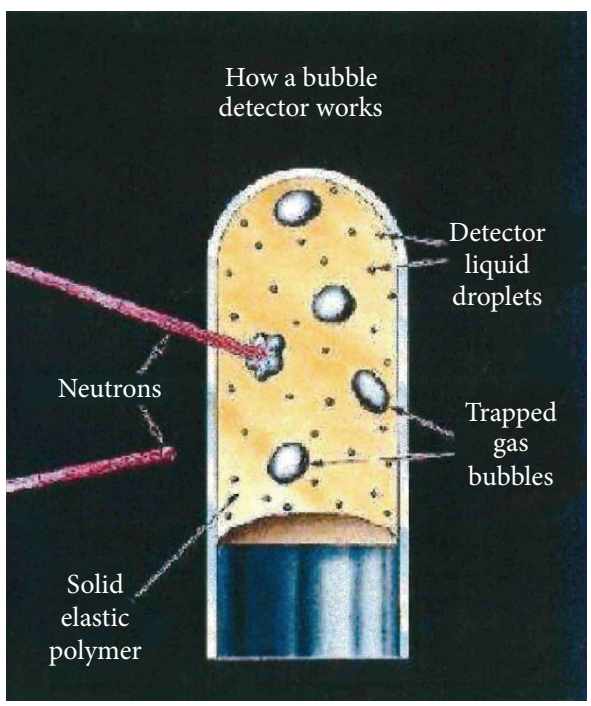

FiguRE 7: SDD for neutron's detectors.

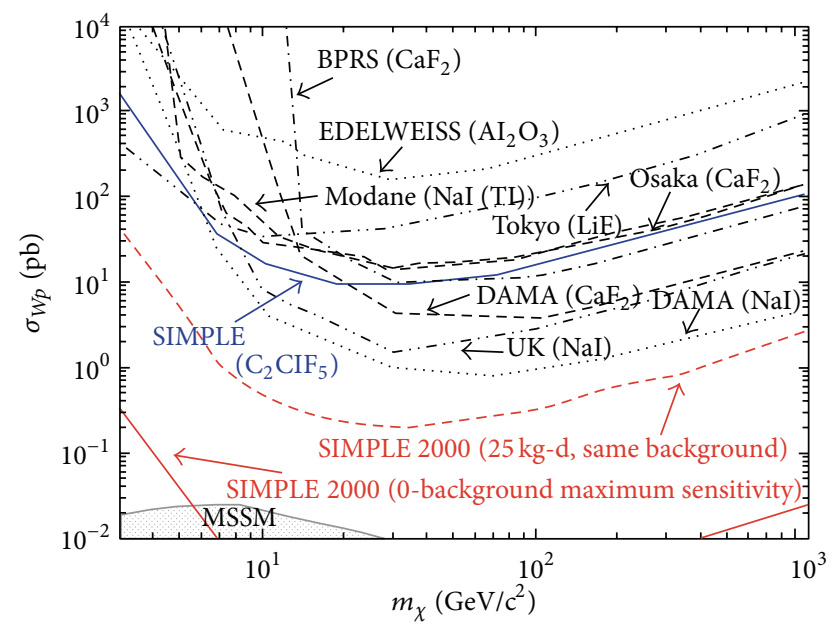

FIGURE 8: SIMPLE 2000: 95\% C.L. limits from only $0.19 \mathrm{~kg}$ days SDD exposure compared with other experiments; the red lines indicate the expected sensitivity after an exposure of $25 \mathrm{~kg}$ days.

(2) The simplicity of the mechanical construction, also for large size detectors, and therefore low cost are an advantage.

(3) The very interesting possibility of counting multiple neutron interactions and hence subtract the neutron background (the interaction length of a neutron is of the order of $(6-20) \mathrm{cm}$ in liquid freon) is an advantage. The double or triple interaction in the same frame can be used statistically to evaluate the number of events with a single interaction due to neutrons.

(4) The possibility of distinguishing the spin-dependent interaction of WIMP from spin-independent by changing the liquid used is an advantage.

(5) Only for the Geyser, the reset of the detector is automatic and has a very short time (few seconds).

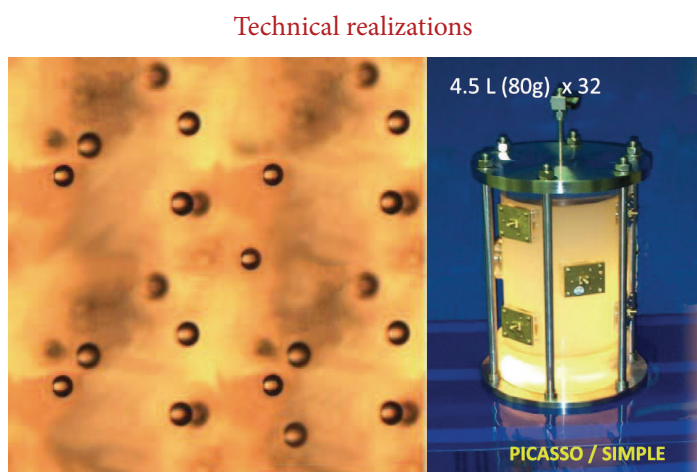

Droplet detectors

$\cdot 150 \mu \mathrm{m}$ superheated droplets of $\mathrm{C}_{4} \mathrm{~F}_{10}$ dispersed in polymerised gel

- Droplets superheated at ambient $T$ and $P\left(T_{b}=-1.7^{\circ} \mathrm{C}\right)$

- Bubble explosion recorded by piezoelectric transducers

- Repressurization $(6 b)$ returns bubbles into droplets

- Operating temperature determines energy threshold

FIgURE 9: The PICASSO-droplet detector.

A prototype of the Geyser with a mass of $0.5 \mathrm{~kg}$ has been constructed in Milano-Bicocca University and INFN [26, 27].

With reference to Figure 12 the quartz vessel of 0.33 liters is immersed in a water bath and it is surrounded by $\mathrm{Cu}$ coils with an internal circulating water at the two fixed temperatures. 6 bars.

It contains freon $\mathrm{C}_{3} \mathrm{~F}_{8}$ around $25^{\circ} \mathrm{C}$ at a pressure of about

The hot freon is separated from the cold freon vapour by the neck of the vessel filled with a buffer liquid (glycol) with thermal capacity greater than that of the water.

In fact in the original Geyser of Hahn and Reist [29] no buffer liquid was used, but we found that it improves greatly the stability of the device.

The temperature of the two regions of water is kept fixed by two thermostats with a precision of 0.1 degrees and the two regions are separated by a loosely fitting rubber washer.

The temperature of the cold vapour was varied between $15^{\circ} \mathrm{C}$ and $21^{\circ} \mathrm{C}$.

Everything is surrounded by a cylindrical vessel of plexiglass of thickness of $1.5 \mathrm{~cm}$, filled with a water/glycol mixture.

In order that the flask undergoes only a small overpressure with respect to the water an automatic pressure equalizer using rubber membranes is used. LEDs.

The freon is illuminated by diffuse light, coming from

To summarize, the Geyser is essentially a vessel constituted by a "flask" containing the overheated liquid (e.g., some kind of freon) and a "neck" (containing partially a separation liquid and partially the freon vapour).

The scattered ions, after an interaction with a neutral particle like a neutron or a WIMP, deposit their energy in very small regions (size of the order of 0.05-0.1 micron).

In these conditions a bubble can grow and reach a few $\mathrm{mm}$ of radius (well visible). 


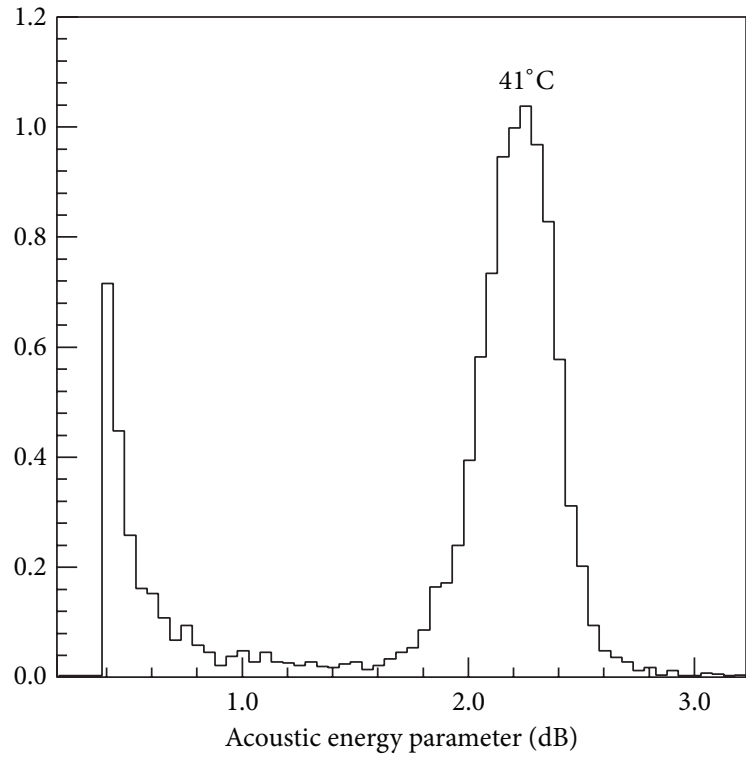

(a)

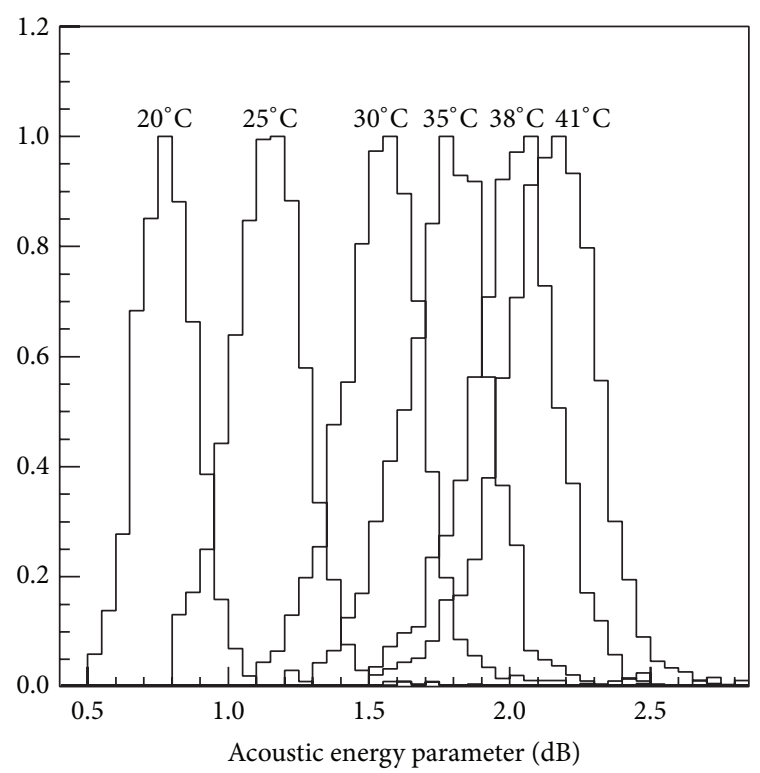

(b)

FIGURE 10: Distribution of the acoustic energy parameter recorded in calibrations with neutrons from Am-Be source; neutron recoils show up an ion peak; this peak is well separated from acoustic and electronic noise (a) and shifts with increasing temperature to larger signal intensities (b).

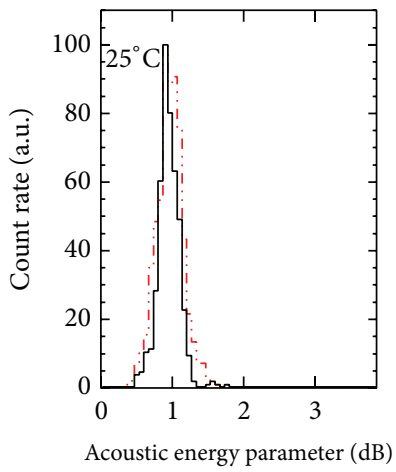

(a)

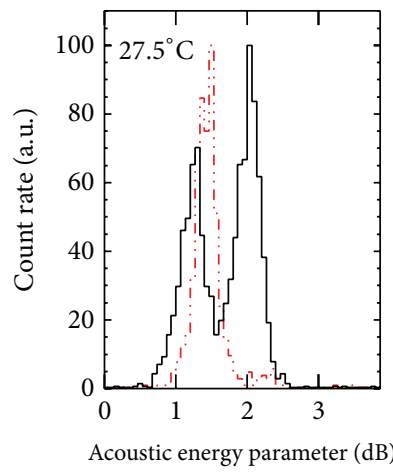

(b)

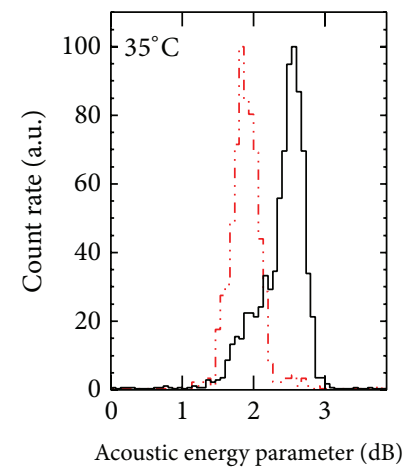

(c)

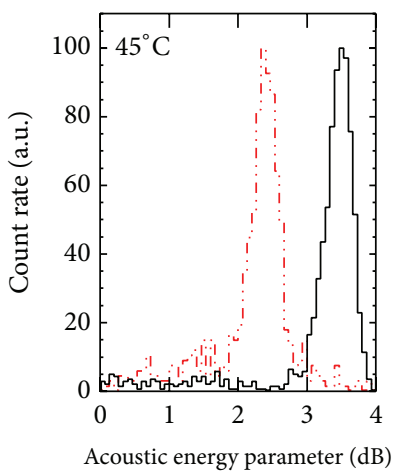

(d)

FIGURE 11: Distribution of the acoustic energy parameter obtained with $\alpha$ decays as a function of temperature; the dotted red histograms indicate the location of recoil events obtained with a neutron source; at low temperature the two peaks coincide, and at higher temperature a second peak appears on the high side for the $\alpha$.

Two professional digital cameras monitor in a continuous way at 50 frames per second (fps) the volume in the freon vessel.

Some pixels undergo a change of luminosity when a bubble is generated.

At this point a trigger is launched and a stream of pictures is registered (between -50 and +50 frames starting from the trigger); in Figure 14 the evolution of a typical bubble observed in our detector is shown.

The time sequence (period $=20 \mathrm{~ms}$ ) starts in the bottom of this figure (right hand), where it is possible to see a small bubble; the sequence continues toward the left and passes to the third line (right); the bubble increases its volume and reaches the surface of the liquid freon (second line); here it produces a small Geyser (left side of the second line); in the first line the passage of the bubble in the lower layers of glycol is shown.

A local energy release, due to, for instance, a recoiling ion induced by a neutron or by a WIMP interaction, can produce a vapour bubble which can grow (if over a threshold in energy) to visible size. This vapour bubble rises in the liquid and pushes up part of the liquid in the neck. When equilibrium pressure is reached the hot vapour in the top of the vessel recondenses and the liquid is recovered into the main volume. The system returns to equilibrium after a few seconds and it is ready to record new events. The system does not require external intervention or recompression. 


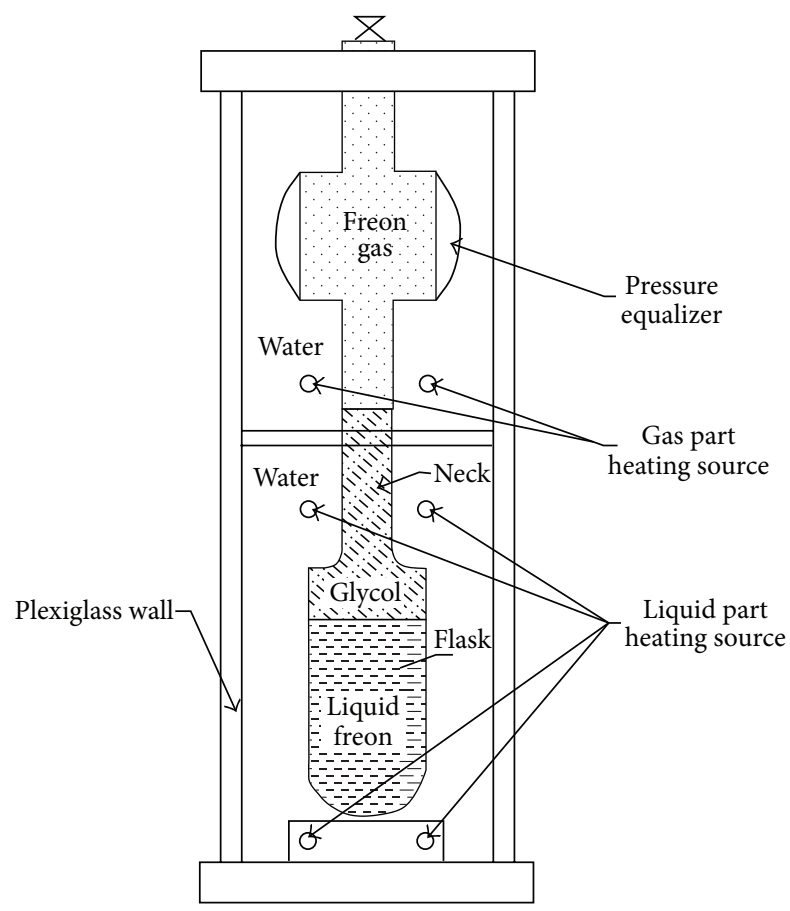

FIGURE 12: Sketch of a vertical section of the Geyser.

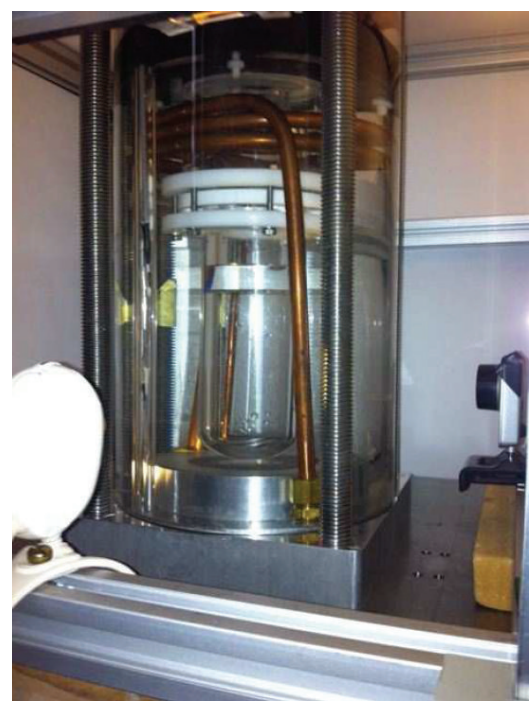

FiguRE 13: Internal Geyser's view.

The degree of superheat applied must exclude the detection of minimum ionizing particles (electrons and $\gamma$ rays) and on the contrary it must allow the detection with high efficiency of the recoiling ions.

A small prototype $(0.3 \mathrm{~L})$ of this kind of detector has been realized in Milano-Bicocca University and INFN [26, 27].

For the future the MOSCAB group wants to construct a bigger detector $(27 \mathrm{~L})$ which could be competitive with other experiments and with other techniques and work in the Laboratory of the Gran Sasso (INFN).

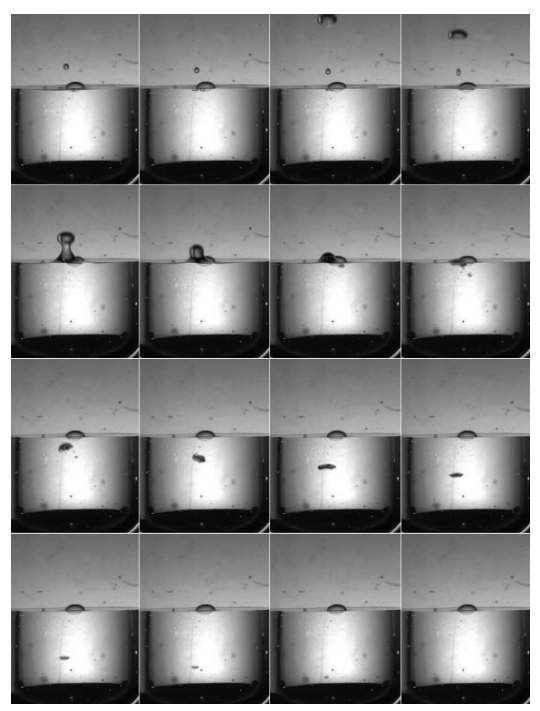

FIGURE 14: Evolution of a bubble.

The transition from a small detector to a very big one requires a lot of new technologies, for the mechanical support, the thermostats, the quartz's vessel, the trigger, the dramatic reduction of the impurities contained in the materials surrounding the detector, and so forth.

However, in particular, information on the spindependent interaction with protons is poor. On the contrary, fluorine based detectors offer excellent opportunities in this field.

The PICASSO and now PICO groups have produced a detailed proposal for the construction of a big detector $(0.5$ tons) [28]. The expected sensitivity for one-year run ( 0.5 tons per year) with a supposed 0 background is shown in Figures 5 and 6.

The future of this kind of detectors depends on the possibilities of extrapolating the data from small prototypes to a very big detector and from the existing residual background in the LNGS and SNOLAB underground laboratories.

\section{Conclusion}

In this short review I have shown that detectors based on the superheated liquid technique have played a relevant role in the search of dark matter. The new generation of ton scale detectors is complementary and competitive with projects employing other techniques. Hopefully, in a few years the mystery of the dark matter will be revealed.

\section{Conflict of Interests}

The author declares that there is no conflict of interests regarding the publication of this paper.

\section{References}

[1] D. A. Glaser, "Some effects of ionizing radiation on the formation of bubbles in liquids," Physical Review, vol. 87, no. 4, p. 665, 1952. 
[2] V. Zacek, "Search for dark matter with moderately superheated liquids," Il Nuovo Cimento A, vol. 107, no. 2, pp. 291-298, 1994.

[3] J. Collar, "Superheated microdrops as cold dark matter detectors," Physical Review D, vol. 54, no. 2, pp. R1247-R1251, 1996.

[4] L. Baudis, "Direct dark matter searches-rapporteur talks," in Proceedings of the New Opportunities in the Physics Landscape at CERN, CERN, May 2009.

[5] F. Seitz, "On the theory of the bubble chamber," Physics of Fluids, vol. 1, no. 1, pp. 2-13, 1958.

[6] G. Jungman, M. Kamionkowski, and K. Griest, "Supersymmetric dark matter," Physics Reports, vol. 267, pp. 195-373, 1996.

[7] E. Behnke, T. Benjamin, S. J. Brice et al., "Direct measurement of the bubble nucleation energy threshold in a $\mathrm{CF}_{3} \mathrm{I}$ bubble chamber," Physical Review D, vol. 88, no. 2, Article ID 021101(R), 2013.

[8] J. I. Collar, J. Puibasset, T. A. Girard, D. Limagne, H. S. Miley, and G. Waysand, "Prospects for SIMPLE 2000: a largemass, low-background superheated droplet detector for WIMP searches," New Journal of Physics, vol. 2, pp. 14.1-14.14, 2000.

[9] R. Bernabei, P. Belli, F. Cappella et al., "New results from DAMA/LIBRA," European Physical Journal C, vol. 67, no. 1-2, pp. 39-49, 2010.

[10] P. Benetti, R. Acciarri, F. Adamo et al., "First results from a dark matter search with liquid argon at $87 \mathrm{~K}$ in the Gran Sasso underground laboratory," Astroparticle Physics, vol. 28, no. 6, pp. 495-507, 2008.

[11] E. Aprile and T. Doke, "Liquid xenon detectors for particle physics and astrophysics," Reviews of Modern Physics, vol. 82, no. 3, pp. 2053-2097, 2010.

[12] D. S. Akerib, M.J. Attisha, C.N. Bailey et al., "Characterization, performance, and future advanced analysis of detectors in the cryogenic dark matter search (CDMS-II)," Nuclear Instruments and Methods in Physics Research A: Accelerators, Spectrometers, Detectors and Associated Equipment, vol. 559, no. 2, pp. 387-389, 2008.

[13] E. Behnke, J. I. Collar, P. S. Cooper et al., "Spin-dependent WIMP limits from a bubble cham," Science, vol. 319, no. 5865, pp. 933-936, 2008.

[14] P. Cooper, CERN PH Seminar, July 2012.

[15] M. Ardid, ICTP Trieste, 2013.

[16] A. Sonnenschein, Private communication.

[17] J. Collar, E. Behnke, P. Cooper et al., COUPP-A Proposal to Operate the COUP-60 Bubble Chamber at SNOLAB, 2009.

[18] J. Collar, E. Behnke, P. Cooper et al., COUPP-500 A Proposal for a Ton Scale Bubble Chamber for Dark Matter Detection, 2010.

[19] R. E. Apfel, “The superheated drop detector," Nuclear Instruments and Methods, vol. 162, no. 1-3, pp. 603-608, 1979.

[20] M. Felizardo, "Final analysis and results of the phase II SIMPLE dark matter search," Physical Review Letters, vol. 108, no. 20, Article ID 201302, 2012.

[21] V. Zacek, Proposal for a 0.5 Ton Dark Matter Experiment at SNOLAB, 2012.

[22] Y. N. Martynyuk and N. S. Smirnova, Soviet Physics: Acoustics, vol. 37, p. 376, 1991.

[23] S. Archambault, F. Aubin, M. Auger et al., "New insights into particle detection with superheated liquids," New Journal of Physics, vol. 13, Article ID 043006, 4 pages, 2011.

[24] S. Adrian-Martinez, A. Albert, M. André et al., "Searches for clustering in the time integrated skymap of the ANTARES neutrino telescope," Journal of Cosmology and Astroparticle Physics, vol. 2014, no. 5, article 001, 2014.
[25] M. G. Aartsen, "Evidence for high-energy extraterrestrial neutrinos at the IceCube detector," Science, vol. 342, no. 6161, Article ID 1242856, 2013.

[26] R. Bertoni, F. Chignoli, D. Chiesa et al., "A new technique for direct investigation of dark matter," Nuclear Instruments and Methods in Physics Research A: Accelerators, Spectrometers, Detectors and Associated Equipment, vol. 744, pp. 61-68, 2014.

[27] A. Pullia, in Proceedings of the VILLA OLMO International Conference 2013-ICATPP Conference, September 2013.

[28] V. Zacek, in Proceedings of the VILLA OLMO International Conference 2013-ICATPP Conference, 2013.

[29] B. Hahn and W. W. Reist, Proceedings of the 5th International Conference on High-Energy Physics Nuclear Structure, 1973. 

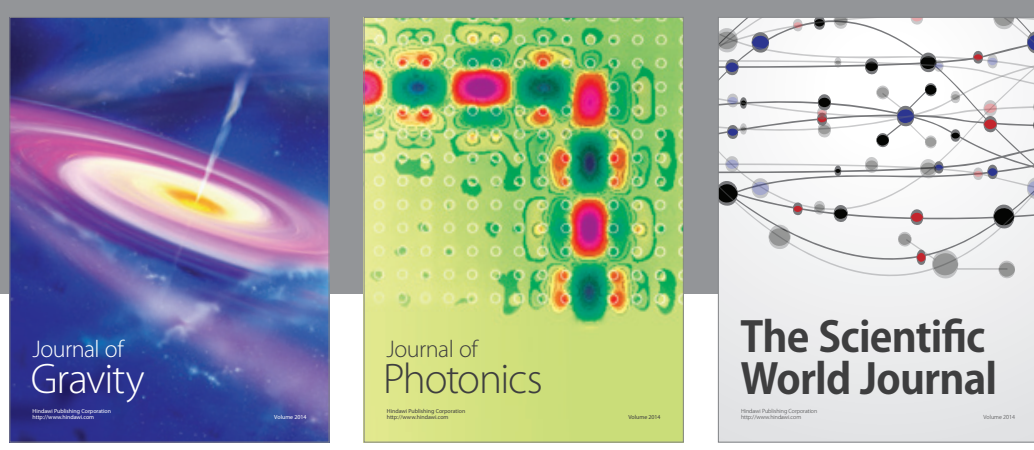

The Scientific World Journal
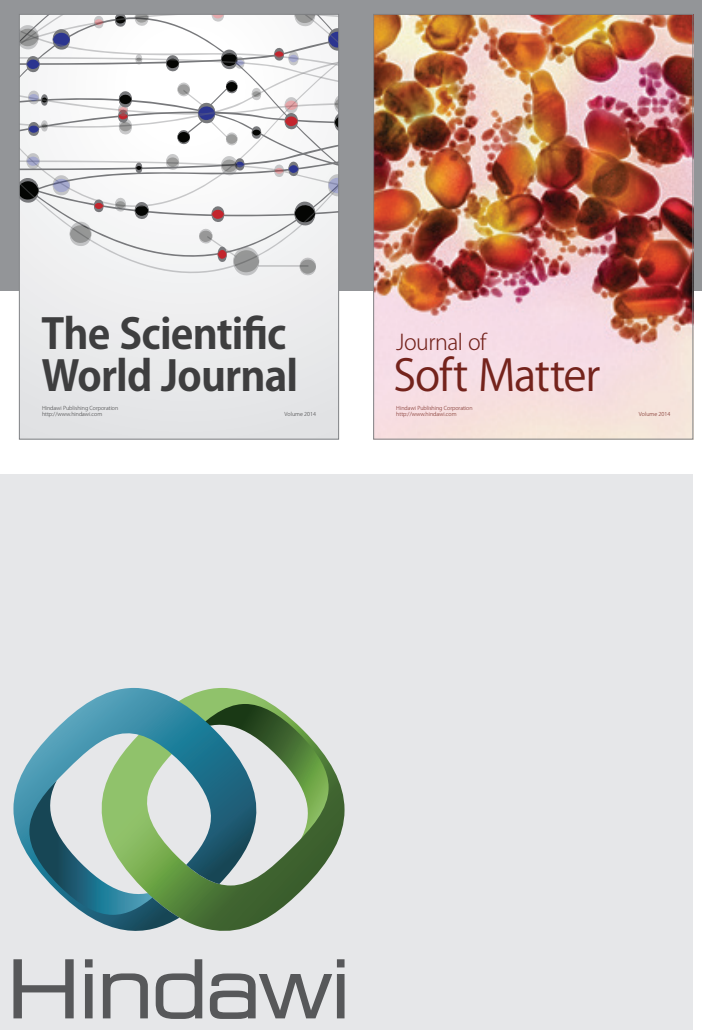

Submit your manuscripts at

http://www.hindawi.com

nternational Journal of

Statistical Mechanics
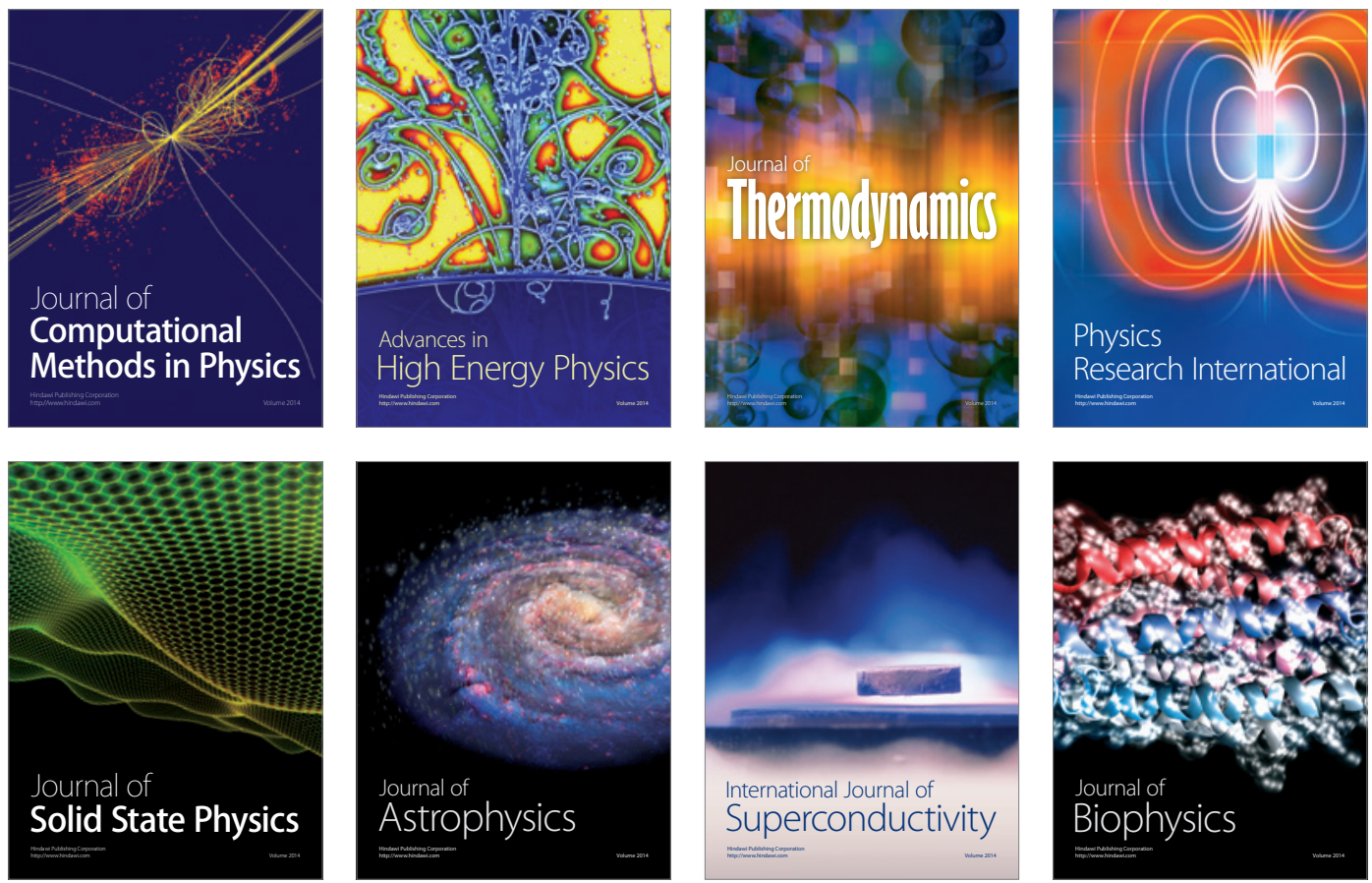
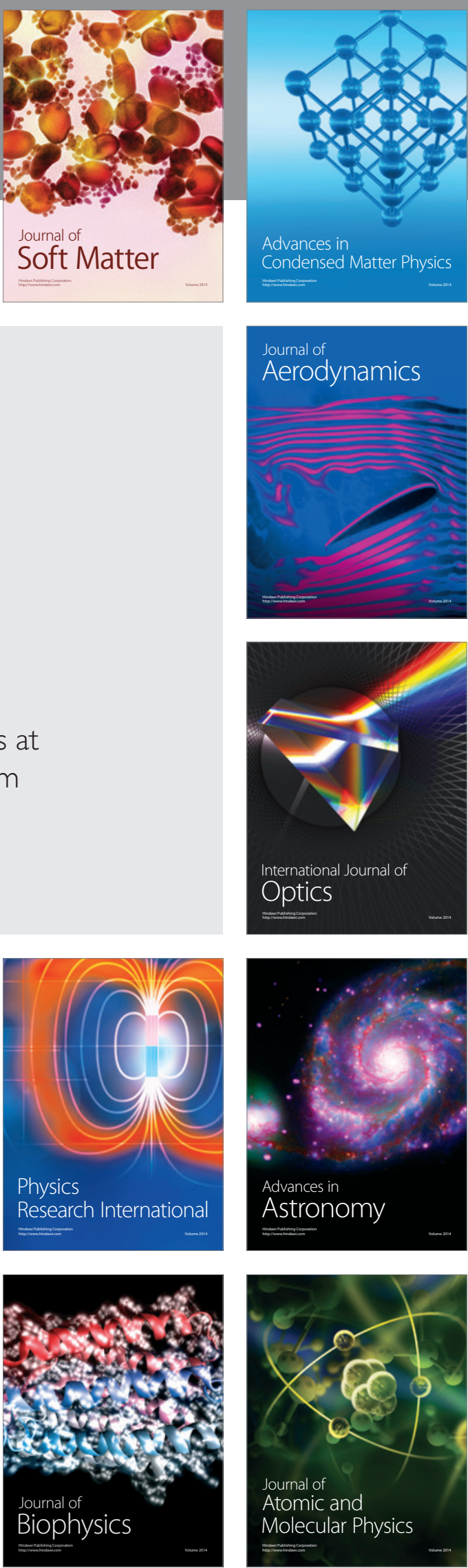